\title{
ENUMERATION OF MIXED GRAPHS ${ }^{1}$
}

FRANK HARARY AND ED PALMER

A mixed graph contains both ordinary and oriented lines. For example the graph in Figure 1 is a mixed graph with two ordinary

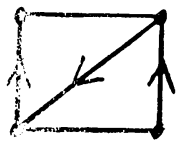

Figure 1

and three oriented lines. An ordinary graph may be regarded as a mixed graph with no oriented lines, and an oriented graph as a mixed graph with no ordinary lines. Further, any digraph may be considered as a mixed graph by changing each symmetric pair of lines to an ordinary line.

Our object is to derive a formula which enumerates mixed graphs on $p$ points with respect to the number of ordinary and oriented lines. For graphical definitions we refer to [4], [5].

Let $m_{p q r}$ be the number of mixed graphs with $p$ points having exactly $q$ oriented lines and $r$ ordinary lines. Then the polynomial $m_{p}(x, y)$ which enumerates mixed graphs with $p$ points according to both the number of ordinary and oriented lines is defined by

$$
m_{p}(x, y)=\sum_{q, r} m_{p q r} x^{q} y^{r}
$$

where

$$
q+r \leqq\left(\begin{array}{l}
p \\
2
\end{array}\right)
$$

From Figure 2, we see that for $p=3$ the formula is

$$
m_{3}(x, y)=1+x+3 x^{2}+2 x^{3}+y+2 x y+3 x^{2} y+y^{2}+x y^{2}+y^{3} .
$$

For the derivation of the formula for $m_{p}(x, y)$, we use a slight modification of Pólya's classical enumeration theorem, [8], in which we use two "figure counting series" rather than one.

Received by the editors October 19, 1965.

1 Work supported in part by the United States Air Force Office of Scientific Research under Grant AF-AFOSR-754-65. 
ENUMERATION OF MIXED GRAPHS

683

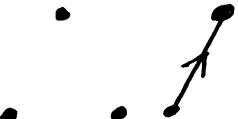

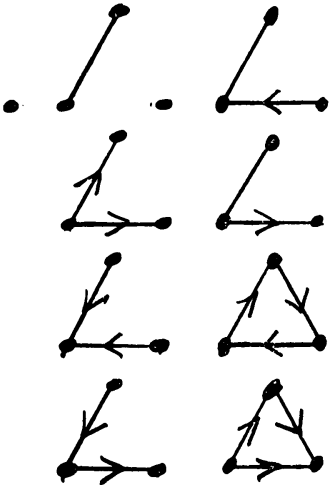
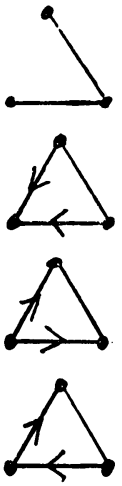
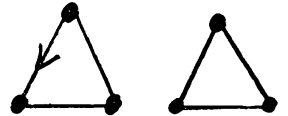

Figure 2

Let $X=\{1,2, \cdots, p\}$ and $Y=\{0,1\}$ and denote the set of ordered pairs $(i, j)$ of distinct elements of $X$ by $X^{[2]}$. The set of functons from $X^{[2]}$ in to $Y$ is denoted as usual by $Y^{X^{[2]}}$. Since each functon $f$ in $Y^{X^{[2]}}$ represents a digraph with say $q$ oriented and $r$ symmetric pairs of lines, $f$ also represents a mixed graph with $q$ oriented lines and $r$ ordinary lines.

The symmetric group $S_{p}$ acting on $X$ induces as in [2] the "reduced ordered pair group" $S_{p}^{[2]}$ acting on $X^{[2]}$. With the identity group $E_{2}$ acting on $Y$, we form the power group $E_{2}^{S_{p}{ }^{[2]}}$ acting on $Y^{X^{[2]}}$; see [6], [7]. Then any two functions $f$ and $g$ in $Y^{X^{[2]}}$ are equivalent with reaspect to the power group $E_{2}^{S_{p}{ }^{[2]}}$ if and only if their mixed graphs are isomorphic.

We may now develop the formula for enumerating mixed graphs.

Let $\alpha$ be any permutation in $S_{p}$ and let $\alpha^{\prime}$ be the permutation in $S_{p}^{[2]}$ induced by $\alpha$. We define the converse of any given cycle in the disjoint cycle decomposition of $\alpha^{\prime}$ as that cycle of $\alpha^{\prime}$ which permutes all ordered pairs $(i, j)$ such that $(j, i)$ is permuted by the given cycle. A cycle of $\alpha^{\prime}$ is called self-converse if $(i, j)$ is permuted by the cycle whenever $(j, i)$ is.

Let $z_{r}$ and $z_{s}$ be distinct cycles of length $r$ and $s$ in the disjoint cycle decomposition of $\alpha$. If $r$ is odd, then $z_{r}$ induces $(r-1) / 2$ pairs of converse cycles of length $r$ in $\alpha^{\prime}$. If $r$ is even, then $z_{r}$ induces $(r-2) / 2$ pairs of converse cycles of length $r$ and one self-converse cycle of length $r$. Together $z_{r}$ and $z_{s}$ induce $d(r, s)$ pairs of converse cycles of length $m(r, s)$, where $d(r, s)$ and $m(r, s)$ are the g.c.d. and 1.c.m. respectively of $r$ and $s$.

It is most convenient to use here the notation of [6] involving the power group of two permutation groups. Suppose $\gamma=\left(\alpha^{\prime} ;(0)(1)\right)$ is 
the permutation in the power group $E_{2}^{S_{p}{ }^{[2]}}$ induced by $\alpha^{\prime}$, and that $\gamma f=f$ for some $f$ in $Y^{x^{[2]}}$. Then the functional values of $f$ are constant on each cycle of $\alpha^{\prime}$. Hence there are exactly three possibilities for the contribution to the mixed graph represented by $f$ by each pair of converse cycles of length $r$ in $\alpha^{\prime}$ :

(1) no lines of either kind occur, or

(2) there are $r$ ordinary lines, or

(3) just one of these two cycles contributes $r$ oriented lines.

Further each self-converse cycle of length $r$ contributes no lines at all or $r / 2$ ordinary lines.

Thus in the terminology of Pólya [8], we see that $(1+2 x+y)^{1 / 2}$ serves as the "figure counting series" to be substituted for all those variables in the cycle index $Z\left(S_{p}^{[2]}\right)$ which specifically correspond to pairs of converse cycles. And $1+y^{1 / 2}$ is the "figure counting series" for the variables corresponding to self-converse cycles. The radical in $(1+2 x+y)^{1 / 2}$ disappears on substitution because converse cycles must occur in pairs. Similarly, the radical in $1+y^{1 / 2}$ disappears because self-converse cycles necessarily have even length.

To effect the appropriate substitutions of these figure counting series, we write the formula from [2] for $Z\left(S_{p}^{[2]}\right)$ with a slight modification of the variables: both $a_{k}$ and $b_{k}$ appear for reasons explained below.

$$
\begin{array}{r}
Z\left(S_{p}^{[2]}\right)=\frac{1}{p !} \sum_{\alpha \in S_{p}}\left\{\prod_{k \text { odd }} a_{k}^{(k-1) j_{k}(\alpha)} \cdot \prod_{k \text { even }}\left(a_{k}^{k-2} b_{k}\right)^{j_{k}(\alpha)} \cdot \prod_{k} a_{k}^{2 k}\left(\begin{array}{c}
j_{k}(\alpha) \\
2
\end{array}\right)\right. \\
\left.\cdot \prod_{1 \leqq r<s \leqq p} a_{m(r, s)}^{2 d(r, s) j_{r}(\alpha) j_{s}(\alpha)}\right\},
\end{array}
$$

where as usual $j_{k}(\alpha)$ is the number of cycles of length $k$ in the disjoint cycle decomposition of the permutation $\alpha$.

For convenience we denote by $Z\left(S_{p}^{[2]},(1+2 x+y)^{1 / 2}, 1+y^{1 / 2}\right)$ the result of substituting $\left(1+2 x^{k}+y^{k}\right)^{1 / 2}$ for each $a_{k}$ in $(2)$ and $1+\left(y^{k}\right)^{1 / 2}$ for each $b_{k}$. This is, of course, the same as substituting $1+2 x^{k}+y^{k}$ for each $a_{k}^{2}$ and $1+y^{k}$ for each $b_{2 k}$. As indicated above, every occurrence of a variable $a_{k}$ will carry an even exponent (since converse cycles come in pairs) and each appearance of a variable $b_{n}$ will have $n$ even (because self-converse cycles have even length).

Then by applying Pólya's theorem [8], the desired counting formula is obtained.

THEOREM. The enumeration polynomial for mixed graphs on p points is given by

$$
m_{p}(\ddot{x}, y)=Z\left(S_{p}^{[2]},(1+2 x+y)^{1 / 2}, 1+y^{1 / 2}\right) .
$$


As an example we give some of the details for $p=3$. First we have the cycle index formulas:

$$
\begin{aligned}
Z\left(S_{3}\right) & =\frac{1}{6}\left(y_{1}^{3}+3 y_{1} y_{2}+2 y_{3}\right), \\
Z\left(S_{3}^{[2]}\right) & =\frac{1}{6}\left(a_{1}^{6}+3 b_{2} a_{2}^{2}+2 a_{3}^{2}\right) .
\end{aligned}
$$

Substituting the figure counting series $(1+2 x+y)^{1 / 2}$ and $1+y^{1 / 2}$, we obtain

$$
\begin{aligned}
m_{3}(x, y) & =\frac{1}{6}\left((1+2 x+y)^{3}+3(1+y)\left(1+2 x^{2}+y^{2}\right)+2\left(1+2 x^{3}+y^{3}\right)\right) \\
& =1+x+3 x^{2}+2 x^{3}+y+2 x y+3 x^{2} y+y^{2}+x y^{2}+y^{3},
\end{aligned}
$$

which agrees pleasantly with the mixed graphs shown in Figure 2.

The counting polynomials $g_{p}(x)$ and $d_{p}(x)$ which enumerate graphs and digraphs were derived in [2], and that for oriented graphs, $o_{p}(x)$, in [3]. We conclude by observing that each of these three polynomials is easily obtained from $m_{p}(x, y)$, which is thus a simultaneous generalization of three previous enumeration formulas:

$$
\begin{aligned}
& d_{p}(x)=m_{p}\left(x, x^{2}\right), \\
& o_{p}(x)=m_{p}(x, 0), \\
& g_{p}(y)=m_{p}(0, y) .
\end{aligned}
$$

For $p=3$, we find from (4) that:

$$
\begin{aligned}
& d_{3}(x)=m_{3}\left(x, x^{2}\right)=1+x+4 x^{2}+4 x^{3}+4 x^{4}+x^{5}+x^{6}, \\
& o_{3}(x)=m_{3}(x, 0)=1+x+3 x^{2}+2 x^{3}, \\
& g_{3}(y)=m_{3}(0, y)=1+y+y^{2}+y^{3} .
\end{aligned}
$$

These are quickly verified by Figure 2 .

A complete digraph has either an oriented line or a symmetric pair of lines joining every pair of points. The digraph in Figure 3 is a complete directed graph on five points with three symmetric pairs and seven oriented lines.

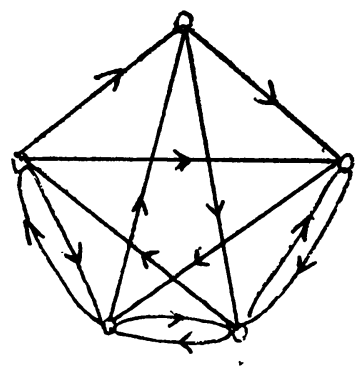

Figure 3 
Let $c_{p q r}$ be the number of complete digraphs with $p$ points having exactly $q$ oriented lines and $r$ symmetric pairs. Then the polynomial $c_{p}(x, y)$ which enumerates complete digraphs with $p$ points according to both the number of oriented lines and symmetric pairs is defined by

$$
c_{p}(x, y)=\sum c_{p q r} x^{q} y^{r}
$$

where $q+r=\left(\begin{array}{l}p \\ 2\end{array}\right)$.

From Figure 4, we see that for $p=3$ the formula is $c_{3}(x, y)=2 x^{3}$ $+3 x^{2} y+x y^{2}+y^{3}$.
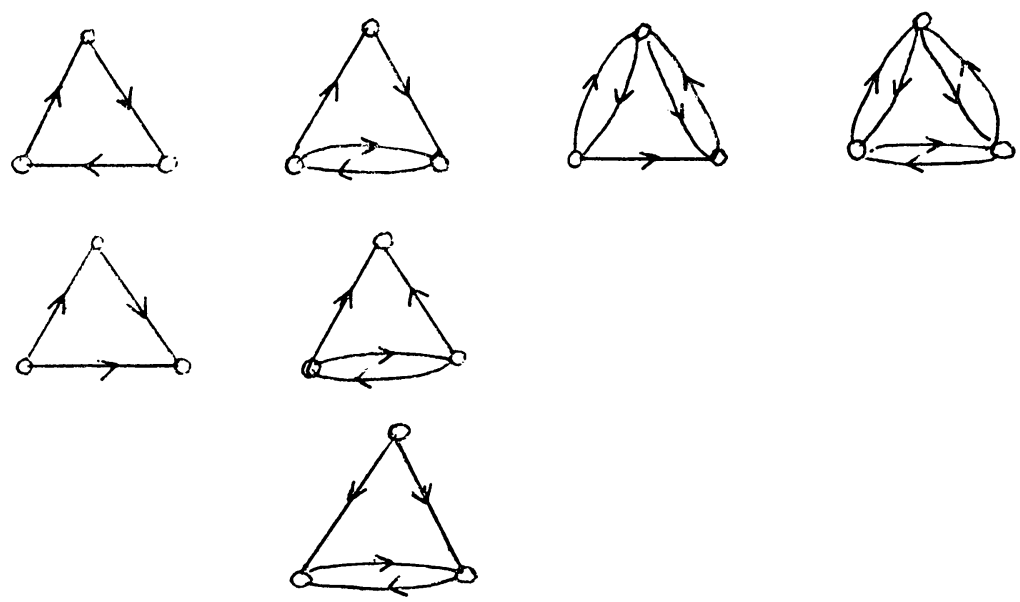

FigURE 4

The enumeration formula for $c_{p}(x, y)$ is easily obtained by modifying the formula for mixed graphs. The integer 1 in each of the two figure counting series $(1+2 x+y)^{1 / 2}$ and $1+y^{1 / 2}$ represents the possibility of having no line joining a pair of points. Since in a complete digraph there is always either an oriented line or a symmetric pair joining a pair of points, the appropriate figure counting series are $(2 x+y)^{1 / 2}$ and $y^{1 / 2}$. Thus we obtain the following corollary.

COROLlaRy. The enumeration polynomial for complete digraphs on $p$ points is given by

$$
c_{p}(x, y)=Z\left(S_{p}^{[2]}, 2 x+y^{1 / 2}, y^{1 / 2}\right) .
$$

An immediate consequence of this corollary is that the number $t_{p}$ of tournaments on $p$ points is

$$
t_{p}=c_{p}(x, 0)
$$


a result previously obtained by Davis [1].

The total number $c_{p}$ of complete digraphs, regardless of the number of oriented lines or symmetric pairs, is

$$
c_{p}=c_{p}(1,1) .
$$

For example, Figure 4 shows that $c_{3}=7$.

Using the formula (2), we obtain the following expression for $c_{p}$.

$$
c_{p}=\frac{1}{p !} \sum_{\alpha \in S_{p}} 3^{e(\alpha)}
$$

where

$$
e(\alpha)=\sum_{k=1}^{p}\left\{\left[\frac{k-1}{2}\right] j_{k}(\alpha)+k\left(\begin{array}{c}
j_{k}(\alpha) \\
2
\end{array}\right)\right\}+\sum_{1 \leqq r<s \leqq p} d(r, s) j_{r}(\alpha) j_{s}(\alpha) .
$$

The first five values of $c_{p}$ are:

\begin{tabular}{l|lllll}
$p$ & 1 & 2 & 3 & 4 & 5 \\
$c_{p}$ & 1 & 2 & 7 & 42 & 582
\end{tabular}.

\section{REFERENCES}

1. R. L. Davis, Structure of dominance relations, Bull. Math. Biophys. 16 (1954), 131-140.

2. F. Harary, The number of linear, directed, rooted and connected graphs, Trans. Amer. Math. Soc. 78 (1955), 445-463.

3. - The number of oriented graphs, Michigan Math. J. 4 (1957), 221-224.

4. - Combinatorial problems in graphical enumeration, Chapter 6 in Applied Combinatorial Mathematics, E. F. Beckenbach, ed., New York, 1964; pp. 185-217.

5. F. Harary, R. Norman and D. Cartwright, Structural models: an introduction to the theory of directed graphs, Wiley, New York, 1965.

6. F. Harary and E. M. Palmer, The power group of two permutation groups. Proc. Nat. Acad. Sci., U.S.A. 54 (1965), 680-682.

7. - - The power group enumeration theorem, J. Combinatorial Theory, 1 (1966) (to appear).

8. G. Pólya, Kombinatorische Anzahlbestimmungen für Gruppen, Graphen and chemische Verbindungen, Acta Math. 68 (1937), 145-254.

The University of Michigan 